\title{
Consensus on Stereotactic Body Radiation Therapy for Small-Sized Hepatocellular Carcinoma at the 7th Asia-Pacific Primary Liver Cancer Expert Meeting
}

\author{
Zhao-Chong Zeng $^{a}$ Jinsil Seong ${ }^{b}$ Sang Min Yoon ${ }^{c}$ \\ Jason Chia-Hsien Cheng ${ }^{d}$ Ka-On Lam $^{e}$ Ann-Shing Lee ${ }^{f}$ \\ Ada Law ${ }^{g}$ Jian-Ying Zhang ${ }^{\text {a }}$ Yong Hu$^{\text {a }}$ \\ a Department of Radiation Oncology, Zhongshan Hospital, Fudan University, Shanghai, \\ China; ${ }^{b}$ Department of Radiation Oncology, Yonsei Cancer Center, Yonsei University \\ College of Medicine, Yonsei University Health System, Seoul, and ' Department of Radiation \\ Oncology, Asan Liver Center, Asan Medical Center, University of Ulsan College of Medicine, \\ Seoul, Korea; d Department of Radiation Oncology, National Taiwan University Hospital, \\ Taipei, Taiwan; ${ }^{e}$ Department of Clinical Oncology, Queen Mary Hospital, Li Ka Shing Faculty \\ of Medicine, The University of Hong Kong, ${ }^{f}$ Department of Clinical Oncology, Tuen Mun \\ Hospital, and 9 Department of Oncology, Pamela Youde Nethersole Eastern Hospital, \\ Hong Kong, SAR, China
}

\section{Keywords}

Hepatocellular carcinoma - Stereotactic body radiation therapy · Survival ·

Radiation-induced liver disease

\begin{abstract}
Background: Stereotactic body radiation therapy (SBRT) is an advanced technique of external beam radiation therapy that delivers large ablative doses of radiation. In the past decade, many cancer centers have adopted SBRT as one mode of radically treating small-sized hepatocellular carcinoma (HCC), based on encouraging clinical outcomes. SBRT thus seems reasonable as first-line treatment of inoperable HCC confined to the liver. However, most of the clinical studies to date have been retrospective in nature, with key issues still under investigation. Summary: The above-mentioned publications were subjected to scrutiny, fueling discussions at the 7th Asia-Pacific Primary Liver Cancer Expert (APPLE 2016) Meeting on various clinical variables, such as indications for SBRT, therapeutic outcomes, treatment-related toxicities, doses prescribed, and specific techniques. The consensus reached should be of interest
\end{abstract}

J. Seong and Z.-C. Zeng contributed equally to this work as corresponding authors.

Jinsil Seong, MD, PhD

Department of Radiation Oncology, Yonsei Cancer Center

Yonsei University Health System, 50-1 Yonsei-ro, Seodaemun-gu

Seoul 120-752 (Korea)

E-Mail jsseong@yuhs.ac
Zhao-Chong Zeng, MD, PhD

Department of Radiation Oncology, Zhongshan Hospital

Fudan University, 136 Yi Xue Yuan Road

Shanghai 200032 (China)

E-Mail zeng.zhaochong@zs-hospital.sh.cn 
to all professionals active in the treatment of HCC, especially radiation oncologists. Key Messages: SBRT is a safe and effective therapeutic option for patients with small-sized HCC, offering substantial local control, improved overall survival, and low toxicity.

(C) 2017 S. Karger AG, Basel

\section{Introduction}

As diagnostic imaging and screening programs for hepatocellular carcinoma (HCC) have progressed, early-stage or small-sized tumors are being detected with greater frequency, enabling treatment locally via surgical resection, radiofrequency ablation (RFA), or transarterial chemoembolization (TACE). Recent technological advances offer precise and safe radiation delivery to multiple tumors in the body through image guidance. Given that the liver is a parallel organ, relatively high locally ablative radiation doses are tolerated. There is growing evidence for the usefulness of stereotactic body radiation therapy (SBRT) in the management of patients with primary liver cancer. The consensus presented herein was developed on the basis of recent evidence and expert opinions.

\section{Definition}

SBRT is a type of external beam radiation therapy technique requiring special equipment for use in patient positioning and delivery of high-dose radiation to body tumors (excluding the brain). Fewer fractions and sharp dose gradients spare normal tissues. This complex technique relies on the following: (1) stringent control of breathing motion, engaging 4-dimensional (4D) computed tomography (CT) scans to track respiration-induced hepatic movement, (2) extreme precision in patient positioning, and (3) image guidance for radiation delivery [1].

Early-stage HCC is defined as a solitary tumor $\leq 5 \mathrm{~cm}$ in maximum diameter or as multiple nodules ( $\leq 3$ total) measuring $\leq 3 \mathrm{~cm}$ in maximum diameter, without vascular invasion/extrahepatic metastasis and with Child-Pugh A or B hepatic function. Not all tumors of small size are qualified as early stage because intrahepatic recurrences of HCC or Child-Pugh C score may apply [2, 3].

\section{Indications for SBRT}

SBRT may be an effective therapeutic option for early-stage or small-sized HCC, especially if surgical resection or percutaneous ablative therapies are difficult, unfeasible, or rejected. This approach is also used as a salvage treatment for tumor recurrence after local radical therapies or for residual cancer after surgical resection or percutaneous ablative attempts. In addition, SBRT may act as a bridge to liver transplantation or serve as an adjuvant treatment for intrahepatic tumors with incomplete iodized oil retention.

\section{Outcomes of SBRT Use for HCC}

Survival

SBRT has emerged as a promising noninvasive therapy in this setting. A limited number of clinical trials examining SBRT use in patients with HCC have been reported, yielding 3-year 


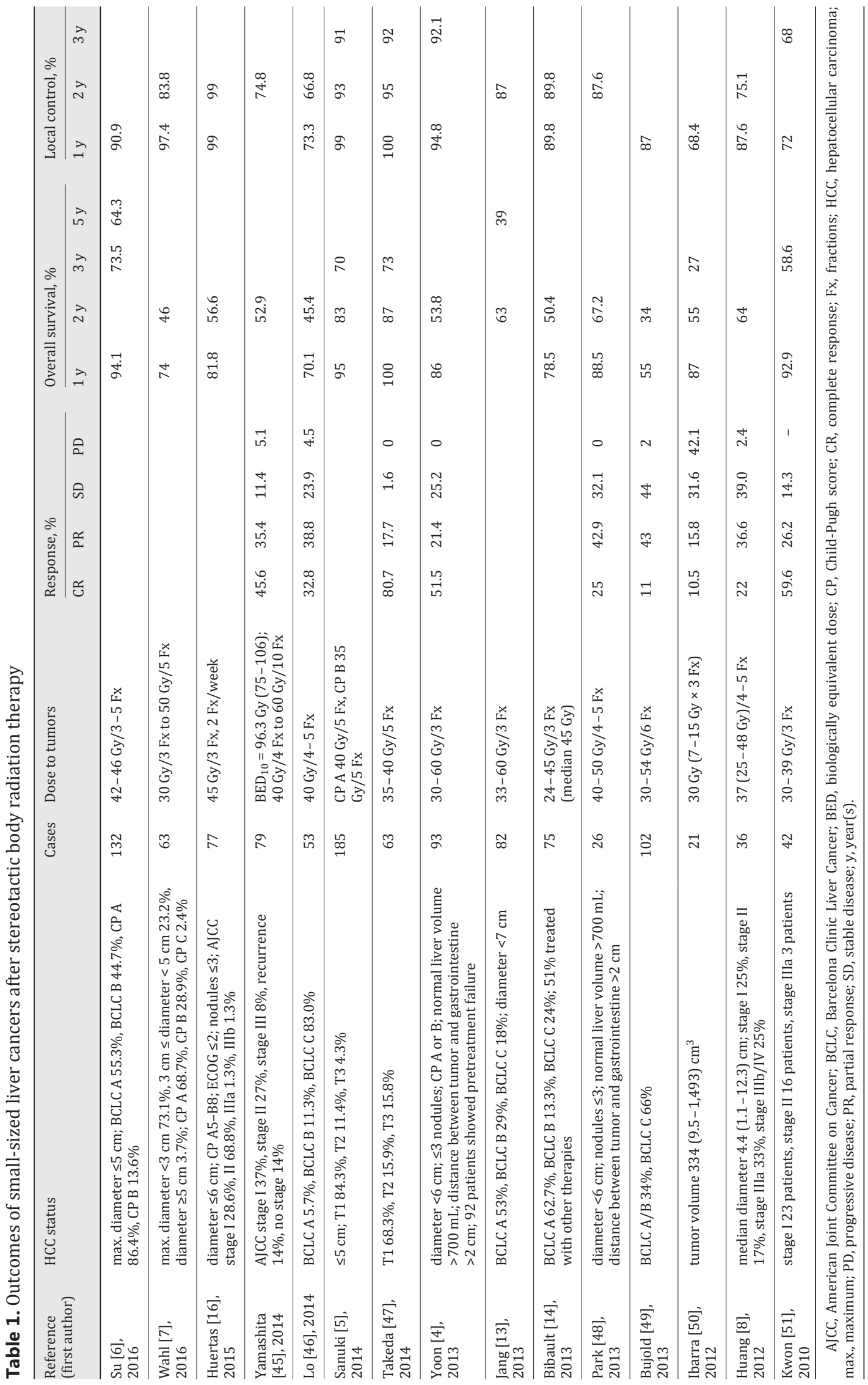


Table 2. Comparison of overall survival for small-sized hepatocellular carcinoma treated with locoregional therapies

\begin{tabular}{lll}
\hline Treatment modality & 3-year survival & 5-year survival \\
\hline Surgical resection & $75-90 \%[9,52]$ & $40-75 \%[10,52]$ \\
Laparoscopic resection & $70-93 \%[54]$ & $50-71 \%[54]$ \\
Radiofrequency ablation & $54-67.2 \%[53]$ & $40-67.9 \%[10,55]$ \\
Liver transplantation & $65-85 \%[59]$ & $65-80 \%[59]$ \\
Stereotactic body radiation therapy & $54-70 \%[4,5]$ & $64 \%[6]$ \\
\hline
\end{tabular}

Reference 4: the hepatocellular carcinoma was $<6 \mathrm{~cm}$ across its longest diameter, and $\leq 3$ lesions were presented. Reference 5: a single (either solitary or recurrent) hepatocellular carcinoma lesion; unfeasible, difficult, or refusal to undergo other surgery or percutaneous ablative therapies, tumor $\leq 5 \mathrm{~cm}$. Reference 6 : maximum diameter $\leq 5 \mathrm{~cm}$. Reference 10: intrahepatic tumor with single nodule $\leq 5 \mathrm{~cm}$ or up to 3 nodules $<3 \mathrm{~cm}$. Reference 53: up to 2 nodules $<4 \mathrm{~cm}$. Reference 55: up to 3 nodules with a maximum diameter of $5 \mathrm{~cm}$.

overall survival (OS) rates of 54-70\% [4, 5] and a 5-year OS rate of 64\% in small-sized HCC [6]. However, such efforts have been limited by small patient numbers and retrospective inclusion of nonsurgical patients. Published results of several retrospective studies (Table 1) have largely included instances in which surgical resection or percutaneous ablative therapies were difficult, unfeasible, or rejected, as well as some pools of intermediate or advancedstage HCC. Herein, only patients receiving $\leq 10$ fractions for HCC were reviewed.

A total of 224 patients with inoperable, nonmetastatic HCC received treatments at Michigan University Medical Center (RFA, 161; image-guided SBRT, 63). Inverse probability of treatment weighting was applied to adjust for imbalances in treatment assignment. In multivariate analysis, OS rates for RFA (1 year, 69.6\%; 2 years, 52.9\%) and SBRT (1 year, 74.1\%; 2 years, $46.3 \%$ ) did not differ significantly. Although based on retrospective data, SBRT nonetheless appears reasonable as first-line treatment of inoperable HCC, especially for tumors $>2 \mathrm{~cm}$ [7].

With adjustment for presumptive prognostic factors, Huang et al. [8] used a Cox multivariable regression hazard model to compare OS curves of SBRT $(n=36)$ and non-SBRT $(n=$ 138 ) patient groups (hazard ratio $=2.44 ; p=0.005$ ). The authors also compared the 2-year OS rates of SBRT recipients $(n=28)$ and matched controls $(n=28)$ (72.6 vs. $42.1 \% ; p=0.013$ ), concluding that SBRT improved survival in patients with recurrent unresectable HCC.

In Table 2, survival data for patients with small-sized HCC are stratified by locoregional treatment modality. The current options for curative treatment of early-stage or small-sized HCC, namely liver transplantation, surgical resection, TACE, and percutaneous tumor ablation, all conform to established authoritative guidelines (i.e., Milan criteria, Barcelona Clinic Liver Cancer staging) [9]. Five-year survival rates of 50-80\% are thereby achieved [10]. Given comparable outcomes (as shown), SBRT constitutes a viable alternative if such therapies fail or cannot be applied.

SBRT is also a suitable bridging therapy for patients with HCC awaiting liver transplantation. At the University of Rochester Medical Center, successful orthotopic liver transplantation was undertaken following SBRT (median, 6.3 months) in 11 of 18 patients with HCC (21 intrahepatic lesions). Recipients had small-sized HCC and Child-Pugh B or C liver function [11]. Similarly, 10 patients with HCC (11 lesions) underwent SBRT at Baylor University, administered as a bridge to liver transplantation. All patients subsequently received liver transplants [12]. SBRT may enable patients with HCC to remain eligible for curative transplantation pending organ availability. 


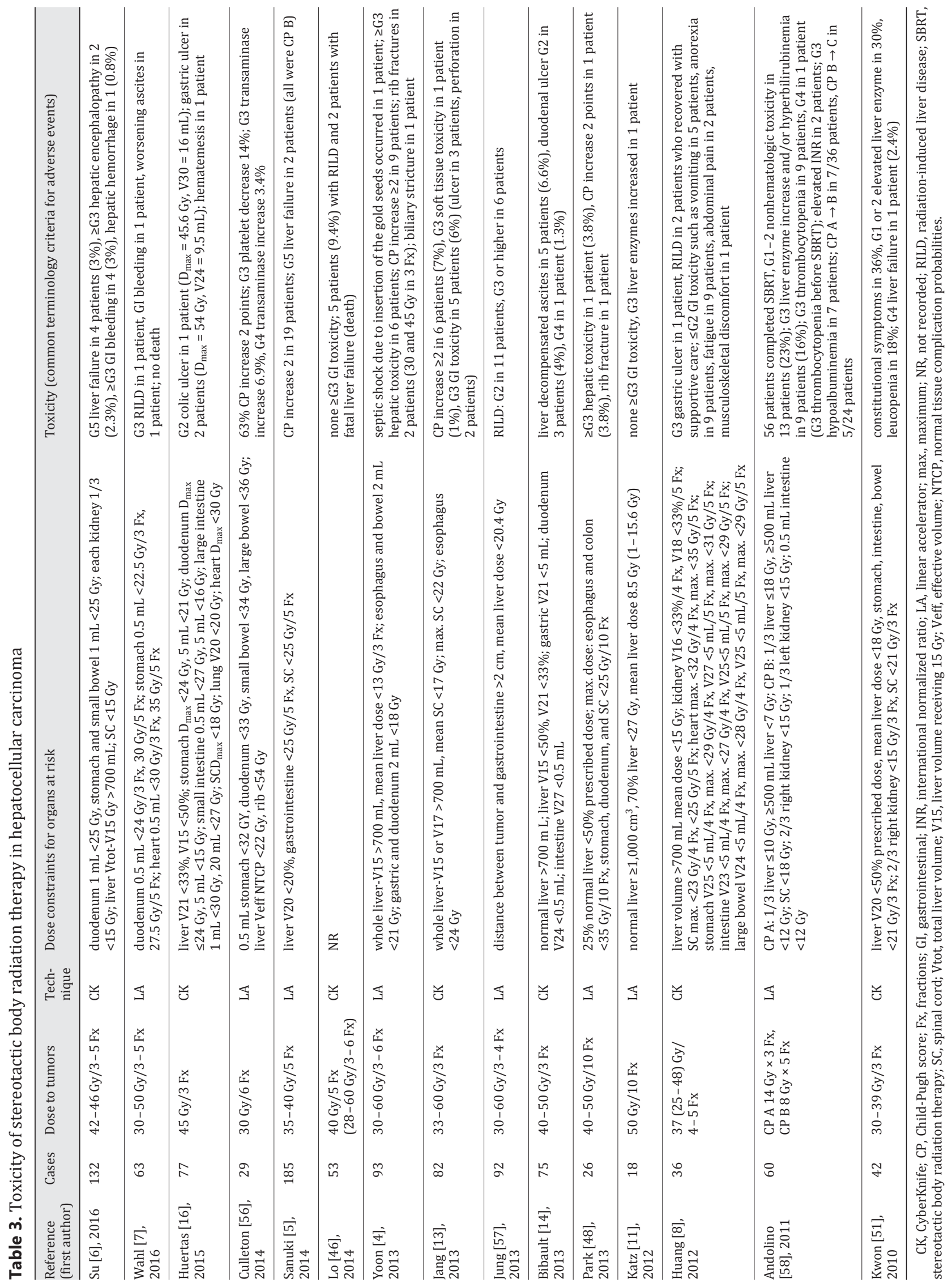


Zeng et al.: Consensus on Stereotactic Body Radiation Therapy for Small-Sized

Hepatocellular Carcinoma at the 7th Asia-Pacific Primary Liver Cancer Expert Meeting

\section{Toxicity}

In terms of acute adverse events, the most common subjective complaints are constitutional symptoms, including fatigue, poor appetite, and nausea. Slight elevation of liver enzymes and leukopenia/thrombocytopenia are the main objective findings. Once SBRT is completed, acute events typically resolve without specific treatment. Nonclassic radiationinduced liver disease (RILD) (liver enzyme elevation, $\geq 2$-point rise in Child-Pugh score) is also common during or after SBRT but is rarely fatal. As shown in Table 3, a summary of 15 reports and 1,063 patients with HCC undergoing SBRT, only 8 patients $(0.8 \%)$ presented with grade 5 liver failure, and most fatalities from liver failure were linked with Child-Pugh B liver function. Caution is thus warranted in patients with Child-Pugh B status, ensuring low dose volumes.

Gastrointestinal (GI) toxicity is another concern, especially if tumors and luminal structures (esophagus, stomach, duodenum, or intestine) are closely situated. GI toxicities of grade 3 or higher were reported in 15 patients $(1.4 \%)$ (Table 3). Other complications, such as rib fracture, chest or abdominal pain, biliary stricture, and musculoskeletal discomfort, were noted occasionally. However, toxicities in general were infrequent and mild.

\section{Radiation Dose}

Tumor Dose

SBRT is an ablative therapy using higher doses to destroy cancer cells. Delivery protocols have varied considerably (24-60 Gy/3-10 fractions). High-dose SBRT may increase local control and improve OS in patients with inoperable HCC. Jang et al. [13] cited respective 2-year local control/OS rates of 100/71,78/64, and 64/30\% ( $p=0.009 / p<0.001)$ at doses $>54,45-54$, and $<45 \mathrm{~Gy}$, all in 3 fractions. SBRT dose was also found to be significantly prognostic of OS $(p=0.005)$ in multivariate analysis, and correlation analysis revealed a positive linear relationship between SBRT dose and local control $(R=0.899 ; p=0.006) / O S(R=0.940$; $p=0.002$ ) at 2 years. Wahl et al. [7] further documented local control rates of 97.4 and $83.8 \%$ at 1 and 2 years, respectively, using a median biologically equivalent dose (BED) of $100 \mathrm{~Gy}$, assuming a $10 \mathrm{~Gy} \alpha / \beta$ ratio. In most instances, $\mathrm{BED}_{10}>80 \mathrm{~Gy}$ was delivered to tumors (Table 1 ). Dose and fractions were usually dictated by tumor size, organs at risk (such as whole liver), and luminal structures nearby. Unfortunately, no evidence has yet emerged to clearly support a minimum or maximum dose of SBRT for HCC. This issue merits further research.

\section{Dose Constraints for Normal Liver}

Su et al. [6], Yoon et al. [4], and Jang et al. [13] from Asia indicated that liver volume receiving 15 Gy (V15) $>700 \mathrm{~mL}$ may lead to grade 3 or fatal RILD at doses of 30-60 Gy/3-6 fractions, but according to Bibault et al. [14] in France, no RILD was encountered at whole normal liver volume $>700 \mathrm{~mL}$ and V15 $<50 \%$, using 40-50 Gy/3 fractions. However, decompensated cirrhosis did develop 3 months later in 5 patients (6.6\%). At normal liver volume $<700 \mathrm{~mL}$ and mean hepatic dose $<15$ Gy (25-48 Gy/4-5 fractions), 2 of the 26 patients studied by Huang et al. [8] developed RILD, but both recovered with supportive care. Still, Katz et al. [11] reported no RILD at normal liver volume $\geq 1,000 \mathrm{~mL}$ and mean dose $<15$ Gy (50 Gy/ 10 fractions). Therefore, at least $700 \mathrm{~mL}$ of normal liver (Child-Pugh A) must receive $<15$ Gy to minimize acute and late liver toxicities [15].

Dose Constraints for the GI Tract and Other Organs at Risk

GI bleeding is the most frequently encountered nonhepatic toxicity following SBRT. With delivery of $<25$ Gy to $1 \mathrm{~mL}$ of duodenum, stomach, or small bowel, the rate of GI bleeding was 
$3.0 \%$ (4/132) [6]. This incidence declined to $1.6 \%$ at doses $<24$ Gy in 3 fractions or 27.5 Gy in 5 fractions per $0.5 \mathrm{~mL}$ [7], and GI ulcers developed in 3.9\% (3/77) [16]. At <21 Gy/3 fractions delivered to $5 \mathrm{~mL}$ of stomach or $<24 \mathrm{~Gy} / 3$ fractions delivered to $0.5 \mathrm{~mL}$ of duodenum, GI toxicity was minimal (grade 2, 3/75; grade 4, 1/75) [14]. Doses $<25,<24$, and $<23$ Gy, respectively delivered to $5 \mathrm{~mL}$ of stomach or intestine in 36 patients were associated with a single instance of gastric ulcer [8]. GI dose constraints should be considered for neighboring hepatic tumors. Compounding the effects of radiation, patients with underlying cirrhosis are at risk of gastroduodenal bleeding or ulcers. Yet, fatal GI toxicity (bleeding, ulceration, or perforation) was rarely reported (Table 3), largely because most doses delivered to $1 \mathrm{~mL}$ of esophagus, stomach, duodenum, or intestine were $<24 \mathrm{~Gy} / 3$ fractions or $<30 \mathrm{~Gy} / 6$ fractions. In other words, fatal GI toxicity was avoidable with appropriate constraints.

No data on radiation-induced injury to the kidney, heart, or spinal cord were available, these organs being remotely situated. In tumors located close to ribs, whole-rib doses should be $<30$ Gy/3 fractions [4].

\section{Combination with Other Therapies}

Jacob et al. [17] retrospectively compared TACE alone and TACE plus SBRT as treatment of HCC in patients with tumor diameters $\geq 3 \mathrm{~cm}$. Local recurrences were significantly reduced and OS improved by combined treatment. Honda et al. [18] also recorded a rise in complete responses upon addition of SBRT to TACE in patients with solitary $\mathrm{HCC} \leq 3 \mathrm{~cm}$, and in patients subjected to SBRT after incomplete response to TACE, the survival outcomes registered by Paik et al. [19] approached those of complete TACE responders or curative measures. SBRT as adjuvant therapy may well improve the efficacy of TACE, but no clinical data at present suggest that TACE improves the efficacy of SBRT in early-stage HCC.

SBRT may also serve as a salvage treatment for residual or recurrent HCC after surgical resection or RFA. However, significant toxicity has resulted from SBRT and sorafenib in combination, with no added benefit. Thus, a regimen of concurrent SBRT and sorafenib is not recommended outside a clinical trial in locally advanced HCC [20]. Patients with hepatitis B virus reactivation and concomitant serum hepatitis B virus DNA elevation or biochemical abnormalities of the liver are advised to receive antiviral therapies prior to SBRT.

\section{SBRT Technique}

\section{Simulation and Target Delineation}

Dynamic contrast-enhanced CT is the preferred imaging modality for treatment planning. If tumors are not well visualized on CT scan, fusion of pretreatment diagnostic magnetic resonance imaging and planning CT images is required. 4D CT simulations are used in patients treated via nonbreathhold technique to gauge gross tumor volume and internal target volume. In SBRT, gross tumor volume, clinical target volume, internal target volume, and planning target volume are stipulated by ICRU Report 62 [21]. Although subclinical extension of HCC is a known phenomenon [22], clinical target volume margin is generally not critical in SBRT, because falloff exposure will likely eradicate any existing microscopic disease. If respiratory gating is applied, internal target volume is the sum of all gross tumor volumes within the predefined gating window. Planning target volume margin is a technique- and center-specific geometrical variation. The entire liver and both kidneys, as well as the spinal cord, duodenum, and stomach, are delineated and should be contoured as organs at risk. 


\section{Immobilization}

Patient immobilization and control of organ motion are crucial for the success of SBRT in this setting. A variety of body frame systems are available, most relying on vacuum cushions, with or without abdominal compression $[23,24]$.

Methods controlling breathing motion include active breathing control (ABC), abdominal compression, respiratory gating, and real-time tumor tracking. ABC involves a modified spirometer, with two pairs of flow monitors and scissor valves to control respiration. Activation is triggered at a predefined lung volume, "freezing" all breathing motion for 15-20 s by closing both valves. ABC-assisted SBRT is quick, generating the smallest planning target volume by comparison. However, pretreatment training is required, and it may be unsuitable for some patients, especially those with reduced lung function [25].

Abdominal compression is a convenient mean of reducing tumor motion by applying a compressive plate or a breath belt during both planning CT and treatment. High levels of forces are required to compress the abdomen [26], and subxiphoid compression is advised for better breathing management, reducing craniocaudal liver motion to within $5 \mathrm{~mm}$ [27]. However, liver deformation and gross tumor volume positional deviation are important consequences of abdominal compression, necessitating rigid liver-to-liver registrations to minimize variations [28].

With respiratory gating, SBRT dose is delivered only in specific phases of the respiratory cycle to avoid unnecessary dosing of normal tissue and underdosing of the target. Treatment time is longer with gating, but it is an acceptable alternative to ABC. The Real-Time Position Management System or the Exac Trac Adaptive Gating System are commonly used.

\section{Image Guidance}

Various imaging modalities are integrated into radiation treatment systems, offering image guidance strategies to produce volumetric and/or planar imaging at the time of treatment delivery and to reduce the negative impact of geometrical changes that may occur [29]. For SBRT in HCC, two available clinical systems enabling tracking of and compensation for patient-specific tumor motion during treatment are the gimbal-based Vero platform and the robot-based CyberKnife [30]. Due to limited tumor visualization in treatment CT scans and X-ray fluoroscopic images, gold fiducial markers are often implanted in liver tissue surrounding tumors to reduce residual setup errors [31], assess breathing motion [32], and track tumor position dynamically [33].

Image-guided radiation delivery is fundamental to SBRT. At present, a number of applications, such as CyberKnife, helical tomotherapy, and volumetric-modulated arc radiotherapy, are SBRT capable [34].

\section{Treatment Plan}

During the past decade, various techniques have been devised for SBRT of HCC. Compared with other methods (3D conformal radiotherapy or intensity-modulated radiation therapy), SBRT plans have very high dose drop speeds around targets and high conformal doses to targets. Tumor volumes are also smaller. Hence, a small grid size (typically $2 \mathrm{~mm}$ ) is recommended for dose calculation [1], and at least 5-9 noncoplanar or coplanar fields should be used for conventional treatment planning [35]. Small-size beam shaping devices may improve conformity of target dose distributions [1]. In some protocols, tissue heterogeneity corrections are included in dose calculations (RTOG1112). Various indices can be applied to evaluate and report plan quality (e.g., conformity index, R50\%, D $2 \mathrm{~cm}$, and heterogeneity index) [1]. The gradient index (same as R50\%) reflects the dose gradient around the planning target volume [36], impacting nearby normal tissue. Hot spots within the target are generally considered a mark of steep dose gradient and are clinically desirable. Considering the differ- 
ences among techniques or protocols, it is essential to report the prescription dose in details (e.g., isodose covering planning target volume to a particular volume).

\section{Post-SBRT Images}

\section{Tumor}

HCC responsive to SBRT appears nonenhanced on imaging, which may be more useful than size reduction in evaluating treatment outcomes during the first 6-12 months (supporting EASL rather than RECIST criteria) [37]. Response rates in hypervascular HCC have increased during follow-up radiologic imaging for as much as to 2 years after completion of SBRT [38].

\section{Liver}

In patients receiving SBRT for HCC, follow-up CT liver dynamics were classifiable as follows: type 1, hyperdensity in all enhanced phases; type 2, hypodensity in arterial and portal phases; and type 3, isodensity in all enhanced phases [39, 40]. Features in the enhancement group (type 1) changed significantly over time, most patients qualifying as Child-Pugh A. In the nonenhancement group (type 3), Child-Pugh B was a significant element $[39,40]$. The incidence of arterial hypervascularity in irradiated hepatic parenchyma may gradually increase for 6 months after SBRT, potentially interfering with accurate assessment of treatment response. Lack of washout on delayed phase in hypervascular areas helps distinguish SBRT-related change from residual/recurrent HCC [41]. Focal liver reaction to SBRT (not to be misread as recurrent HCC) has a threshold dose of $30 \mathrm{~Gy} / 5$ fractions for Child-Pugh A and 25 Gy/5 fractions for Child-Pugh B [42, 43] or 20 Gy/3 fractions [44]. Of note, uncertainty exists between the imaging changes, the pathological fibrosis, and the serological data. These doses will help predict potential loss of liver tissue after SBRT.

\section{Conclusion}

In conclusion, SBRT is a safe and effective therapeutic option for patients with smallsized HCC, offering substantial local control, improved OS, and low toxicity.

\section{Disclosure Statement}

The authors declare no conflicts of interest.

\section{References}

1 Benedict SH, Yenice KM, Followill D, et al: Stereotactic body radiation therapy: the report of AAPM Task Group 101. Med Phys 2010;37:4078-4101.

2 Han KH, Kudo M, Ye SL, etal: Asian consensus workshop report: expert consensus guideline for the management of intermediate and advanced hepatocellular carcinoma in Asia. Oncology 2011;81(suppl 1):158-164.

3 Yau T, Tang VY, Yao TJ, et al: Development of Hong Kong Liver Cancer staging system with treatment stratification for patients with hepatocellular carcinoma. Gastroenterology 2014;146:1691-1700.e3.

4 Yoon SM, Lim YS, Park MJ, et al: Stereotactic body radiation therapy as an alternative treatment for small hepatocellular carcinoma. PLoS One 2013;8:e79854.

5 Sanuki N, Takeda A, Oku Y, et al: Stereotactic body radiotherapy for small hepatocellular carcinoma: a retrospective outcome analysis in 185 patients. Acta Oncol 2014;53:399-404.

6 Su TS, Liang P, Lu HZ, et al: Stereotactic body radiation therapy for small primary or recurrent hepatocellular carcinoma in 132 Chinese patients. J Surg Oncol 2016;113:181-187. 
Zeng et al.: Consensus on Stereotactic Body Radiation Therapy for Small-Sized

Hepatocellular Carcinoma at the 7th Asia-Pacific Primary Liver Cancer Expert Meeting

7 Wahl DR, Stenmark MH, Tao Y, et al: Outcomes after stereotactic body radiotherapy or radiofrequency ablation for hepatocellular carcinoma. J Clin Oncol 2016;34:452-459.

8 Huang WY, Jen YM, Lee MS, et al: Stereotactic body radiation therapy in recurrent hepatocellular carcinoma. Int J Radiat Oncol Biol Phys 2012;84:355-361.

9 Korean Liver Cancer Study Group (KLCSG); National Cancer Center, Korea (NCC): 2014 KLCSG-NCC Korea Practice Guideline for the Management of Hepatocellular Carcinoma. Gut Liver 2015;9:267-317.

10 de Lope CR, Tremosini S, Forner A, et al: Management of HCC. J Hepatol 2012;56(suppl 1):S75-S87.

11 Katz AW, Chawla S, Qu ZH, et al: Stereotactic hypofractionated radiation therapy as a bridge to transplantation for hepatocellular carcinoma: clinical outcome and pathologic correlation. Int J Radiat Oncol Biol Phys 2012; 83:895-900.

12 O'Connor JK, Trotter J, Davis GL, et al: Long-term outcomes of stereotactic body radiation therapy in the treatment of hepatocellular cancer as a bridge to transplantation. Liver Transpl 2012;18:949-954.

13 Jang WI, Kim MS, Bae SH, et al: High-dose stereotactic body radiotherapy correlates increased local control and overall survival in patients with inoperable hepatocellular carcinoma. Radiat Oncol 2013;8:250.

14 Bibault JE, Dewas S, Vautravers-Dewas C, et al: Stereotactic body radiation therapy for hepatocellular carcinoma: prognostic factors of local control, overall survival, and toxicity. PLoS One 2013;8:e77472.

15 Sawrie SM, Fiveash JB, Caudell JJ: Stereotactic body radiation therapy for liver metastases and primary hepatocellular carcinoma: normal tissue tolerances and toxicity. Cancer Control 2010;17:111-119.

16 Huertas A, Baumann AS, Saunier-Kubs F, et al: Stereotactic body radiation therapy as an ablative treatment for inoperable hepatocellular carcinoma. Radiother Oncol 2015;115:211-216.

17 Jacob R, Turley F, Redden DT, et al: Adjuvant stereotactic body radiotherapy following transarterial chemoembolization in patients with non-resectable hepatocellular carcinoma tumours of $\geq 3 \mathrm{~cm}$. HPB (Oxford) 2015; 17:140-149.

18 Honda Y, Kimura T, Aikata H, et al: Stereotactic body radiation therapy combined with transcatheter arterial chemoembolization for small hepatocellular carcinoma. J Gastroenterol Hepatol 2013;28:530-536.

19 Paik EK, Kim MS, Jang WI, et al: Benefits of stereotactic ablative radiotherapy combined with incomplete transcatheter arterial chemoembolization in hepatocellular carcinoma. Radiat Oncol 2016;11:22.

20 Brade AM, Ng S, Brierley J, et al: Phase 1 trial of sorafenib and stereotactic body radiation therapy for hepatocellular carcinoma. Int J Radiat Oncol Biol Phys 2016;94:580-587.

21 ICRU: Prescribing, recording and reporting photon beam therapy (supplement to ICRU Report No. 50). ICRU Report No. 62, 1999.

22 Wang MH, Ji Y, Zeng ZC, et al: Impact factors for microinvasion in patients with hepatocellular carcinoma: possible application to the definition of clinical tumor volume. Int J Radiat Oncol Biol Phys 2010;76:467-476.

23 Hansen AT, Petersen JB, Høyer M: Internal movement, set-up accuracy and margins for stereotactic body radiotherapy using a stereotactic body frame. Acta Oncol 2006;45:948-952.

24 Case RB, Sonke JJ, Moseley DJ, et al: Inter- and intrafraction variability in liver position in non-breath-hold stereotactic body radiotherapy. Int J Radiat Oncol Biol Phys 2009;75:302-308.

25 Eccles C, Brock KK, Bissonnette JP, et al: Reproducibility of liver position using active breathing coordinator for liver cancer radiotherapy. Int J Radiat Oncol Biol Phys 2006;64:751-759.

26 Heinzerling JH, Anderson JF, Papiez L, et al: Four-dimensional computed tomography scan analysis of tumor and organ motion at varying levels of abdominal compression during stereotactic treatment of lung and liver. Int J Radiat Oncol Biol Phys 2008;70:1571-1578.

$27 \mathrm{Hu}$ Y, Zhou YK, Zeng ZC, et al: 4D-CT scans reveal reduced magnitude of respiratory liver motion achieved by different abdominal compression plate positions in patients with intrahepatic tumors undergoing helical tomotherapy. Med Phys 2016;43:4335.

28 Eccles CL, Dawson LA, Moseley JL, et al: Interfraction liver shape variability and impact on GTV position during liver stereotactic radiotherapy using abdominal compression. Int J Radiat Oncol Biol Phys 2011;80:938-946.

29 Brock KK: Imaging and image-guided radiation therapy in liver cancer. Semin Radiat Oncol 2011;21:247-255.

30 Sothmann T, Blanck 0, Poels K, et al: Real time tracking in liver SBRT: comparison of CyberKnife and Vero by planning structure-based $\gamma$-evaluation and dose-area-histograms. Phys Med Biol 2016;61:1677-1691.

31 Wunderink W, Méndez Romero A, Seppenwoolde Y, et al: Potentials and limitations of guiding liver stereotactic body radiation therapy set-up on liver-implanted fiducial markers. Int J Radiat Oncol Biol Phys 2010;77: 1573-1583.

32 Heinz C, Gerum S, Freislederer P, et al: Feasibility study on image guided patient positioning for stereotactic body radiation therapy of liver malignancies guided by liver motion. Radiat Oncol 2016;11:88.

33 Kitamura K, Shirato H, Shimizu S, et al: Registration accuracy and possible migration of internal fiducial gold marker implanted in prostate and liver treated with real-time tumor-tracking radiation therapy (RTRT). Radiother Oncol 2002;62:275-281.

34 Wang PM, Hsu WC, Chung NN, et al: Feasibility of stereotactic body radiation therapy with volumetric modulated arc therapy and high intensity photon beams for hepatocellular carcinoma patients. Radiat Oncol 2014; 9:18.

35 Liu R, Buatti JM, Howes TL, et al: Optimal number of beams for stereotactic body radiotherapy of lung and liver lesions. Int J Radiat Oncol Biol Phys 2006;66:906-912.

36 Paddick I, Lippitz B: A simple dose gradient measurement tool to complement the conformity index. J Neurosurg 2006;105(suppl):194-201. 
Zeng et al:: Consensus on Stereotactic Body Radiation Therapy for Small-Sized

Hepatocellular Carcinoma at the 7th Asia-Pacific Primary Liver Cancer Expert Meeting

37 Price TR, Perkins SM, Sandrasegaran K, et al: Evaluation of response after stereotactic body radiotherapy for hepatocellular carcinoma. Cancer 2012;118:3191-3198.

38 Sanuki N, Takeda A, Mizuno T, et al: Tumor response on CT following hypofractionated stereotactic ablative body radiotherapy for small hypervascular hepatocellular carcinoma with cirrhosis. AJR Am J Roentgenol 2013;201:W812-W820.

39 Sanuki-Fujimoto N, Takeda A, Ohashi T, et al: CT evaluations of focal liver reactions following stereotactic body radiotherapy for small hepatocellular carcinoma with cirrhosis: relationship between imaging appearance and baseline liver function. Br J Radiol 2010;83:1063-1071.

40 Kimura T, Takahashi S, Takahashi I, et al: The time course of dynamic computed tomographic appearance of radiation injury to the cirrhotic liver following stereotactic body radiation therapy for hepatocellular carcinoma. PLoS One 2015; 10:e0125231.

41 Park MJ, Kim SY, Yoon SM, et al: Stereotactic body radiotherapy-induced arterial hypervascularity of nontumorous hepatic parenchyma in patients with hepatocellular carcinoma: potential pitfalls in tumor response evaluation on multiphase computed tomography. PLoS One 2014;9:e90327.

42 Sanuki N, Takeda A, Oku Y, et al: Threshold doses for focal liver reaction after stereotactic ablative body radiation therapy for small hepatocellular carcinoma depend on liver function: evaluation on magnetic resonance imaging with Gd-EOB-DTPA. Int J Radiat Oncol Biol Phys 2014;88:306-311.

43 Takeda A, Oku Y, Sanuki N, et al: Dose volume histogram analysis of focal liver reaction in follow-up multiphasic CT following stereotactic body radiotherapy for small hepatocellular carcinoma. Radiother Oncol 2012; 104:374-378.

44 Jung J, Yoon SM, Cho B, et al: Hepatic reaction dose for parenchymal changes on Gd-EOB-DTPA-enhanced magnetic resonance images after stereotactic body radiation therapy for hepatocellular carcinoma. J Med Imaging Radiat Oncol 2016;60:96-101.

45 Yamashita H, Onishi H, Matsumoto $\mathrm{Y}$, et al: Local effect of stereotactic body radiotherapy for primary and metastatic liver tumors in 130 Japanese patients. Radiat Oncol 2014;9:112.

46 Lo CH, Huang WY, Lee MS, et al: Stereotactic ablative radiotherapy for unresectable hepatocellular carcinoma patients who failed or were unsuitable for transarterial chemoembolization. Eur J Gastroenterol Hepatol 2014;26:345-352.

47 Takeda A, Sanuki N, Eriguchi T, et al: Stereotactic ablative body radiotherapy for previously untreated solitary hepatocellular carcinoma. J Gastroenterol Hepatol 2014;29:372-379.

48 Park JH, Yoon SM, Lim YS, et al: Two-week schedule of hypofractionated radiotherapy as a local salvage treatment for small hepatocellular carcinoma. J Gastroenterol Hepatol 2013;28:1638-1642.

49 Bujold A, Massey CA, Kim JJ, et al: Sequential phase I and II trials of stereotactic body radiotherapy for locally advanced hepatocellular carcinoma. J Clin Oncol 2013;31:1631-1639.

50 Ibarra RA, Rojas D, Snyder L, et al: Multicenter results of stereotactic body radiotherapy (SBRT) for nonresectable primary liver tumors. Acta Oncol 2012;51:575-583.

51 Kwon JH, Bae SH, Kim JY, et al: Long-term effect of stereotactic body radiation therapy for primary hepatocellular carcinoma ineligible for local ablation therapy or surgical resection. Stereotactic radiotherapy for liver cancer. BMC Cancer 2010;10:475.

52 Huang J, Yan L, Cheng Z, et al: A randomized trial comparing radiofrequency ablation and surgical resection for HCC conforming to the Milan criteria. Ann Surg 2010;252:903-912.

53 Feng K, Yan J, Li X, et al: A randomized controlled trial of radiofrequency ablation and surgical resection in the treatment of small hepatocellular carcinoma. J Hepatol 2012;57:794-802.

54 Guro H, Cho JY, Han HS, et al: Current status of laparoscopic liver resection for hepatocellular carcinoma. Clin Mol Hepatol 2016;22:212-218.

55 Lee DH, Lee JM, Lee JY, et al: Radiofrequency ablation of hepatocellular carcinoma as first-line treatment: longterm results and prognostic factors in 162 patients with cirrhosis. Radiology 2014;270:900-909.

56 Culleton S, Jiang H, Haddad CR, et al: Outcomes following definitive stereotactic body radiotherapy for patients with Child-Pugh B or C hepatocellular carcinoma. Radiother Oncol 2014;111:412-417.

57 Jung J, Yoon SM, Kim SY, et al: Radiation-induced liver disease after stereotactic body radiotherapy for small hepatocellular carcinoma: clinical and dose-volumetric parameters. Radiat Oncol 2013;8:249.

58 Andolino DL, Johnson CS, Maluccio M, et al: Stereotactic body radiotherapy for primary hepatocellular carcinoma. Int J Radiat Oncol Biol Phys 2011:81:e447-e453.

59 Mazzaferro V, Bhoori S, Sposito C, et al: Milan criteria in liver transplantation for hepatocellular carcinoma: an evidence-based analysis of 15 years of experience. Liver Transpl 2011;17(suppl 2):S44-S57. 